\title{
Article
}

\section{Primitive Chain Network Simulations of Conformational Relaxation for Individual Molecules in the Entangled State}

\author{
Yuichi MASUBUCHI ${ }^{*, * *}$, Hiroshi WATANABE ${ }^{*}$, Giovanni IANNIRUBERTO ${ }^{* * *}$, \\ Francesco GRECO ${ }^{* * * *}$, and Giuseppe MARRUCCI ${ }^{* * *}$ \\ *Institute for Chemical Research, Kyoto University, Gokasyo, Uji, Kyoto 611-0011, Japan. \\ ${ }^{* *}$ JST-PRESTO, Kyoto 611-0011, Japan. \\ **** Dipartimento di Ingegneria Chimica, Universita degli studi di Napoli “Federico II", \\ Piazzale Tecchio 80-80125 Napoli, Italy \\ ${ }^{* * * *}$ Istituto di Ricerche sulla Combustione, CNR, Piazzale Tecchio 80-80125 Napoli, Italy \\ (Received : March 18, 2008)
}

\begin{abstract}
Conformational relaxation of individual chain molecules in the entangled state has already been investigated by direct observation in fluorescent DNA experiments. Yet, possible connections between the quantity accessible in those experiments and well defined conformational measures have not been established so far. In this study, the relaxation behavior of the individual polymer conformation in the entangled state is simulated through the primitive chain network model, and compared to well known relaxations like that of stress or electric polarization. Inspired by the experiments, we use as a measure of the individual conformation the maximum distance between segments of a given polymer chain, which is often referred to as chain extension, and indicated by the symbol $x$. The relaxation behavior is calculated as auto-correlation function at equilibrium, suitably ensemble averaged to eliminate the noise. It is found that such $x$ relaxation is significantly different from both stress relaxation and end-to-end dielectric relaxation, though the power-law exponent of the longest relaxation time vs. chain length appears to be the same for both the stress and the $x$ measure.

Key Words: Entangled polymers / Primitive chain network simulation / Conformational relaxation / Individual polymer motion
\end{abstract}

\section{INTRODUCTION}

Several researchers following the landmark study by Perkins et $\mathrm{al}^{1)}$ have performed observation of individual DNA molecules with fluorescent microscopy, assuming that DNA dynamics exhibits the universal behavior of ordinary flexible polymers. Indeed, although DNA is known as a rigid polyelectrolyte, it has been reported that under conditions of high ionic strength the observed DNA diffusion can be explained by well established models for flexible and neutral synthetic polymers. ${ }^{1-4)}$ For entangled DNA solutions, both fluorescent microscopy and rheological data have been recently reported, showing consistency between microscopic and macroscopic relaxation times.

Detailed analysis of the relaxation functions or spectra of the observed individual polymer motion in entangled solutions has not been reported, however, probably because of uncertainty in the "measure" of the individual molecular

To whom correspondence should be addressed.

E-mail: mas@scl.kyoto-u.ac.jp, Tel: +81-774-38-3136, Fax: +81-774-38-3139 relaxation in microscopic experiments. Indeed, in the experiments, DNA is stained uniformly along its contour and observed as projected 2D image in the plane of the high sensitive fluorescence detector. Consequently, data from the fluorescent intensity matrix on the detected images are used as measures of the molecular conformation, to be compared to the theoretically well-defined molecular measures such as segmental orientation, end-to-end vector, etc. From the experiment, a typical measure of the extension of the polymer chain is the distance between the most separated fluorescence spots, indicated as $x$. It should be noted, however, that no theoretical prediction for $x$ is in fact available. For example, if one assumes that any luminosity (no matter how small) can be detected, in the Gaussian chain the measure $x$ would be infinity at all times during relaxation. For dilute solutions, the measure $x$ is usually assumed to be equivalent to the end-toend distance, and some consistency with the dumbbell and other coarse-grained chain models has been found. ${ }^{6)}$ On the other hand, for entangled solutions, consistency with the tube model remains an open problem. For instance, Teixeira et al. ${ }^{5)}$ reported that in entangled solutions the relaxation of $x$ from 
stretched states cannot be explained by Rouse dynamics in the short time region, while the longest relaxation time is found to be consistent with the rheological data.

In this study we perform primitive chain network simulations ${ }^{7)}$ and compare relaxation functions for both ensemble and individual-molecule measures (including the $x$ measure used in the fluorescent microscopy experiments). We then discuss the various relaxation functions as well as the effects of possible artifacts.

\section{MODEL AND SIMULATIONS}

We used the primitive chain network model that has been already reported in several publications. ${ }^{7-12)}$ In this section, however, we briefly present the model used in this particular study to eliminate possible confusion among the various versions differing in minor aspects. In the model of the entangled polymer network, each entanglement node has four converging strands consistently with a binary assumption of chain interaction, and the node position $\{\mathbf{R}\}$ in space is determined by a force balance including the tensions of the conversing strands, the friction force against the medium, the osmotic force generated by local density fluctuations, and a random force of thermal origin. Following the sliplink picture of an entanglement node, transport of monomers from one strand to the next along the polymer chain is monitored through the monomer number $\{n\}$ in each strand, and is determined by a $1 \mathrm{D}$ kinetic equation accounting for the same physico-chemical forces appearing in the node dynamics. In addition to node motion and monomer transport, entanglement and disentanglement among chains by reptation and fluctuation are modeled as creation and destruction of sliplinks at the chain ends. Hence the number $\{Z\}$ of strands in each chain, or equivalently the entanglement number, is an additional set of stochastic state variables.

We choose the mean equilibrium strand length $a$ as unit of length, which is written as $a=\sqrt{n_{0}} b$, where $b$ is the length of monomers (actually Kuhn segments), and $n_{0}$ is the mean equilibrium value of the monomer number in a strand. The unit of energy is set to $k T$, and the unit of time is $\tau=a^{2} \zeta / 6 k T$ where $\zeta$ is the friction coefficient of the network node.

The relaxation equations for $\{\mathbf{R}\}$ and $\{n\}$ in nondimensional form are:

$$
\begin{gathered}
\dot{\mathbf{R}}=\frac{1}{2} \sum_{i}^{4} \frac{\mathbf{r}_{i}}{n_{i}}-\frac{1}{3} \nabla \mu+\mathbf{F} \\
\frac{\dot{n}}{d}=\frac{r_{i}}{n_{i}}-\frac{r_{i-1}}{n_{i-1}}-\frac{1}{3} \nabla \mu+f
\end{gathered}
$$

where $\mathbf{r}$ is the strand vector and $\mu$ is the chemical potential derived from the free energy $F$ given by

$$
F=\left\{\begin{array}{lll}
\varepsilon\left(\frac{\phi}{\langle\phi\rangle}-1\right)^{2} & \text { for } & \phi>\langle\phi\rangle \\
0 & \text { for } & \phi \leq\langle\phi\rangle
\end{array}\right.
$$

where $\phi$ is the local segment density, and $\langle\cdots\rangle$ means the average over the system. $\mathbf{F}$ and $f$ are Gaussian random forces representing thermal motion in $3 \mathrm{D}$ and $1 \mathrm{D}$, respectively. The local monomer density $d$ along the chain is given by:

$$
d=\frac{1}{2}\left(\frac{n_{i}}{r_{i}}+\frac{n_{i-1}}{r_{i-1}}\right)
$$

The fluctuation of $\{Z\}$ due to creation and destruction of the nodes at the chain ends is triggered by monomer number at the chain ends. If the number of monomers at the end of a chain is less than a certain minimum, the neighboring node is destroyed (disentanglement event), and $Z$ decreases in both chains belonging to that node. On the contrary, if the number of monomers in a chain end exceeds a certain maximum, a new node is created by hooking a randomly selected surrounding strand, and this event causes an increment of $Z$. The monomer number window where topology is preserved is:

$$
0.5<n<1.5
$$

Simulations were performed with monodisperse linear chains consisting of $\langle Z\rangle=4.9,9.6$ and 19.2 strands dispersed in a simulation box of size $12^{3}$ with the usual periodic boundary conditions. Strand density in the box was set to 10 .

\section{MEASURES}

As macroscopic measures, we focus on the usual stress and electrical polarization. The nondimensional stress tensor $\mathbf{T}$ is given by:

$$
\mathrm{T}_{\alpha \beta}=3\left\langle\frac{r_{\alpha} r_{\beta}}{n}\right\rangle
$$

The electrical polarization $\mathbf{P}$ for type-A polymers ${ }^{13)}$ is obtained as:

$$
P_{\alpha}=\left\langle\sum_{z} r_{\alpha}\right\rangle
$$

It should be noted that DNA is not a type-A polymer, yet it is worth considering the $\mathbf{P}$ relaxation for comparison with other 
measures of chain conformation since the dielectric relaxation of type-A polymers has been thoroughly investigated also in the context of well-defined molecular pictures. ${ }^{13)}$

In relation to the chain extension $x$ measure employed in DNA experiments ${ }^{5,6,14,15)}$, we obtain such measure from our simulations in different ways, as shown in Fig. 1. Fig. 1 (a) indicates chain conformation in $3 \mathrm{D}$ space and, from the current position of each node, the simulation can readily calculate the maximum distance between the nodes of a chain in 3D space. Fig. 1 (a) also shows the 2D projection of the $3 \mathrm{D}$ conformation on the $\mathrm{x}-\mathrm{y}$ plane (simulating the fluorescence detector of the high-sensitive CCD camera used in the DNA experiments) and, similarly to the case of the 3D conformation, we can readily obtain a different $x$ measure as the maximum node separation in 2D. In Figs. 1 (b) to (d), in the results of the $2 \mathrm{D}$ projection we examine the effect of the digital detector resolution. We mimic different resolutions by setting different square meshes on the $x-y$ plane, and count the number of the chain nodes falling in each detector cell, thus obtaining an intensity matrix. From the intensity matrix we calculate the maximum separation between the cells having a nonzero signal from the chain. From the intensity matrix we also obtain the angle $\theta$ between the longer axis of the gyration tensor and a coordinate axis.

The relaxation functions of the above defined measures under equilibrium conditions were obtained as autocorrelation functions from the Green-Kubo formula. The relaxation functions for the bulk measures, e.g., stress and (a)

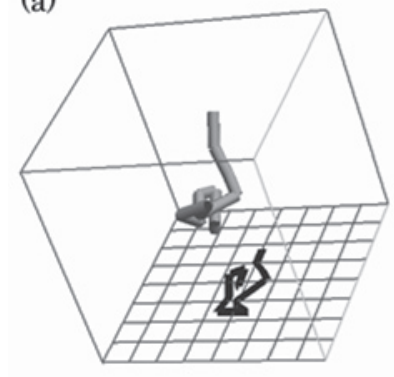

(b)

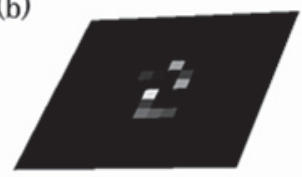

(c)

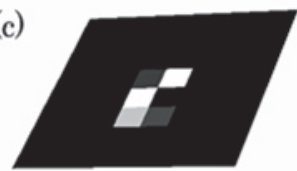

(d)

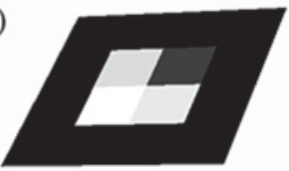

Fig. 1. A typical snapshot of the simulation shown as (a) 3D structure and its projection onto the detector plane. (b), (c), (d) correspond to intensity matrices constructed from the $2 \mathrm{D}$ projection with a detection cell size of $0.5,1.0,2.0$, respectively. electrical polarization, are calculated by using time averages of the signal recorded for a time period larger than $10^{3}$ the observed longest relaxation time. The relaxation functions for individual chain measures are calculated as ensemble averages of the auto-correlation functions of 100 chains. Each individual auto-correlation function was obtained over a time period 20 times longer than the longest relaxation time. Note that, unless differently specified, the $x$ relaxations presented in the following section were calculated for the projection onto the detector plane with a cell dimension of $a^{2}$ (unity in nondimensional variables), corresponding to Fig. 1 (c).

\section{RESULTS AND DISCUSSION}

Figure 2 shows relaxation functions for the measures defined in the previous section, i.e., shear stress, electrical polarization, the $x$ measure, and $\cos \theta$. As previously observed $^{13)}$, stress relaxation is faster than dielectric relaxation, and becomes equal to the square of the dielectric relaxation at large times. On the other hand, the relaxation functions of the $x$ measure, and of $\cos \theta$, which are close to one another, are different from both stress and dielectric relaxation. This result is not trivial because, at first sight, the $x$ and the dielectric relaxation are calculated from apparently similar measures of the indivudual chain conformation. Indeed, for dilute solutions it has been reported that the $x$ relaxation from a stretched conformation can be consistently described by using a dumbbell model where $x$ is assumed to coincide with the

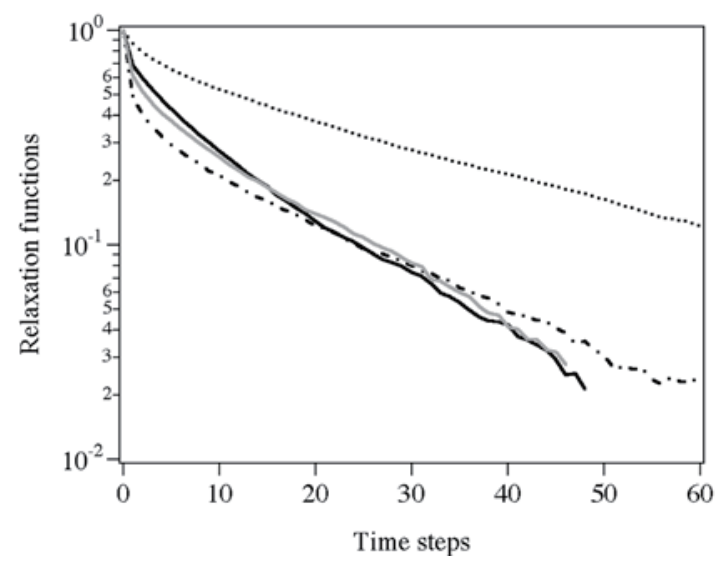

Fig. 2. Relaxation functions for electrical polarization (dotted line), stress (broken line), chain extension measure $x$ (solid line), and chain orientation $\cos \theta$ (hatched line). 
end-to-end vector of the dumbbell. ${ }^{14)}$

Figure 2 also shows that in the long time region all relaxation functions can be described by a single exponential function, from which we can extract the longest relaxation time by a least square fitting. Figure 3 shows the molecular weight dependence of the relaxation time for the $x$ relaxation. The stress relaxation time is also plotted for comparison. It is apparent that the molecular weight scaling behavior of both relaxation times is similar. This means that the $x$ relaxation is not dominated by fast relaxation modes of polymer dynamics like local Rouse relaxation, though the exact relationship between the two relaxation functions is at present unknown.

Figure 4 shows the influence on the $x$ relaxation of the detection cell size, which corresponds to the space resolution in the experiments. Within the examined range (from 0.5 to 2.0 in nondimensional units, see Fig.1), the relaxation function

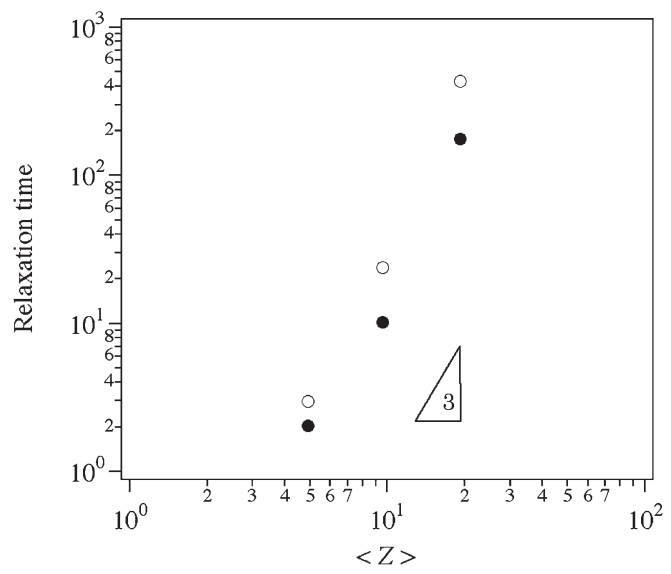

Fig. 3. Relaxation time extracted from the relaxation functions for stress (open symbols) and chain extension measure $x$ (closed symbols) vs. the molecular weight.

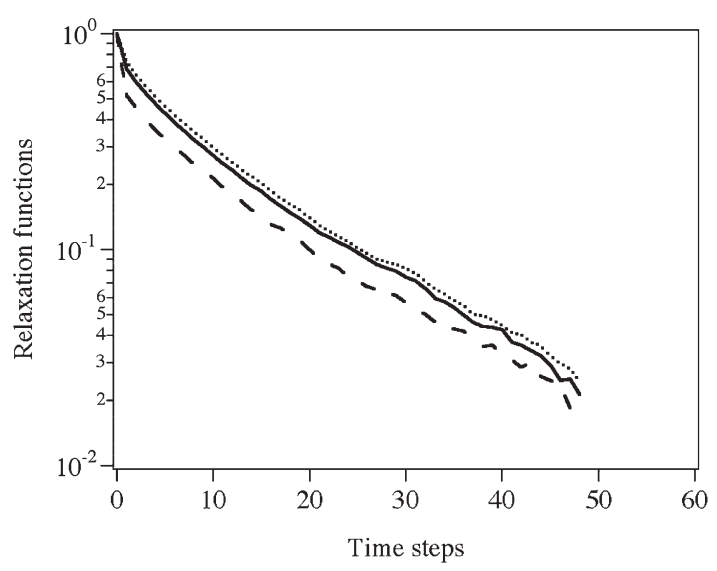

Fig. 4. Relaxation of the chain extension measure $x$ for detector resolutions of 0.5 (dotted line), 1.0 (solid line), and 2.0 (dashed line). is not sensitive to the cell size. It should be noted that similar results were obtained for all the examined chain lengths. To avoid complications on experimental details, we here ignore possible effects of smearing and/or defocusing ${ }^{16)}$, to be carefully examined in future work.

Figure 5 shows the relaxation function which is obtained for the following different definitions of the $x$ measure: i) maximum distance between position of the nodes in 3-D space, ii) same for the 2-D projection of the chain, and iii) that obtained by cell detection. It is apparent that all these relaxation functions are similar to one another, and the effect of cell detection is limited.

Figure 6 shows the effect on $x$ relaxation of the sampling chain number used in the ensemble average. Chain numbers were 10,100 and 1000 . The quality of the data is fair for 100 chains as compared to that for 1,000 chains, while the curve

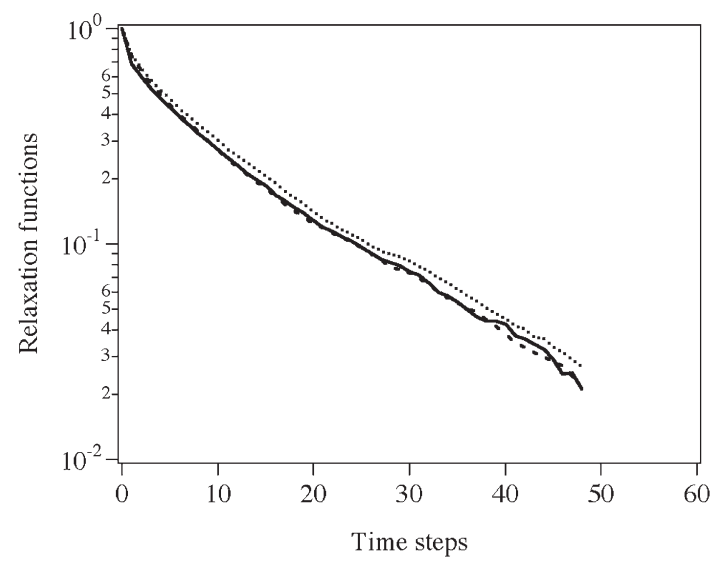

Fig. 5. Relaxation of the chain extension measure for various definitions of $x$ : Maximum separation among segments in 3D space (dashed line), in the 2D projection (dotted line), and that obtained from the intensity matrix (solid line).

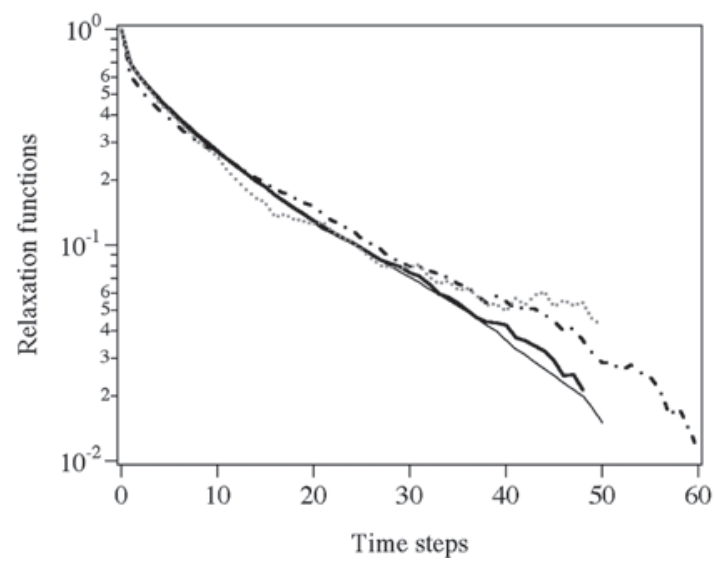

Fig. 6. Relaxation of the chain extension measure $x$ for various ensemble average chain numbers: 10 (dotted line), 100 (solid line), 1000 (thin line). The auto-correlation of $x$ for a single chain with a time average is also shown with a dash-dotted line. 
for 10 chains shows a significant deviation, from which a wrong relaxation time would be estimated. Figure 6 also shows the auto-correlation of the $x$ measure for a single chain, calculated as time average by using a time trace of 100,000 time steps. Since for a single auto-correlation function a time trace of 1,000 steps is used, the single chain calculation with 100,000 time steps would be expected to be similar in statistics to the ensemble average of 100 chains. We find, conversely, that the quality of the data for the single chain calculation comes out much worse.

\section{CONCLUSIONS}

Relaxation functions of various conformational measures for entangled polymers were calculated with primitive chain network simulations. With reference to fluorescence observation of individual DNA molecules, the maximum distance between chain segments in a given polymer chain (or the maximum distance between detector cells receiving signals from the chain segments) was considered. It was found that the relaxation behavior of such distances differs significantly from both stress and dielectric relaxation, while the longest relaxation time obeys a similar scaling behavior with molecular weight. Possible ambiguities in definition of the individual chain measure, the effect of the detector resolution, and the sensitivity to number of sampled chains were examined, and shown to be negligible. The effect on the individual chain measure of flow and deformation is now being examined and the results will be reported elsewhere.

\section{REFERENCES}

1) Perkins TT, Smith DE, Chu S, Science, 264 (5160), 819 (1994).

2) Matsumoto M, Sakaguchi T, Kimura H, Doi M, Minagawa K, Matsuzawa Y, Yoshikawa K, Journal of Polymer Science Part B-Polymer Physics, 30 (7), 779 (1992).

3) Smith DE, Perkins TT, Chu S, Physical Review Letters, 75 (22), 4146 (1995).

4) Smith DE, Perkins TT, Chu S, Macromolecules, 29 (4), 1372 (1996).

5) Teixeira RE, Dambal AK, Richter DH, Shaqfeh ESG, Chu S, Macromolecules, 40 (7), 2461 (2007); e.c. 40 (9), 3514 (2007).

6) Shaqfer ESG, Journal of Non-Newtonian Fluid Mechanics, 130, 1 (2005).

7) Masubuchi Y, Takimoto JI, Koyama K, Ianniruberto G, Marrucci G, Greco F, Journal of Chemical Physics, 115 (9), 4387 (2001).

8) Masubuchi Y, Ianniruberto G, Greco F, Marrucci G, Journal of Chemical Physics, 119 (13), 6925 (2003)

9) Masubuchi Y, Ianniruberto G, Greco F, Marrucci G, Modelling and Simulation in Materials Science and Engineering, 12 (3), S91 (2004).

10) Masubuchi $Y$, Watanabe $H$, Ianniruberto $G$, Greco F, Marrucci $G$, Nihon Reoroji Gakkaishi, 32 (4), 197 (2004).

11) Masubuchi Y, Nihon Reoroji Gakkaishi, 34 (5), 275 (2006).

12) Furuichi $K$, Nonomura $C$, Masubuchi $Y$, Ianniruberto $G$, Greco $F$, Marrucci G, Nihon Reoroji Gakkaishi, 35 (2), 73 (2007).

13) Watanabe H, Progress in Polymer Science, 24 (9), 1253 (1999).

14) Perkins TT, Smith DE, Chu S, Science, 264 (5160), 822 (1994).

15) Larson RG, Perkins TT, Smith DE, Chu S, Physical Review E, 55, 1794 (1997).

16) Baxter WT, Davidenko, JM, Loew LM, Wuskell JP, Jalife JJ, Annals of Biomedical Engineering, 25 (4), 713 (1997). 